\title{
Decoupling of ion conductivity from segmental dynamics in oligomeric ethylene oxide functionalized oxanorbornene dicarboximide homopolymers
}

\author{
Marisa Adams ${ }^{1}$, Victoria Richmond ${ }^{1}$, Douglas Smith ${ }^{1}$, Yangyang Wang ${ }^{2}$, Fei Fan ${ }^{4}$, \\ Alexei P. Sokolov* ${ }^{3,4}$, Dean A. Waldow*1 \\ ${ }^{1}$ Department of Chemistry, Pacific Lutheran University, Tacoma, WA 98447 USA \\ ${ }^{2}$ Center for Nanophase Materials Sciences, Oak Ridge National Laboratory, Oak Ridge, TN 37831 USA \\ ${ }^{3}$ Chemical Sciences Division, Oak Ridge National Laboratory, PO Box 2008, Oak Ridge, TN 37831- \\ 6197, USA \\ ${ }^{4}$ Department of Chemistry, University of Tennessee-Knoxville, Knoxville, TN 37996 USA
}

ARTICLE INFO

ABSTRACT

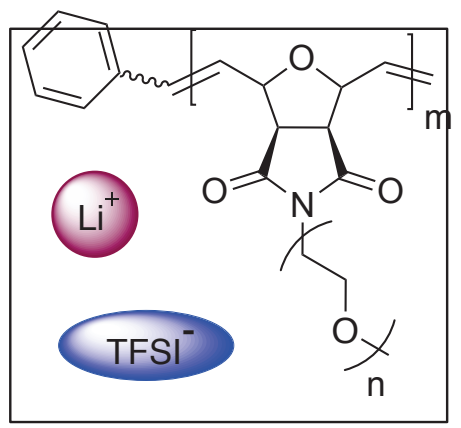

Article history:

Submitted 15 December 2016

Revision Submitted 12 March 2017

Keywords:

Solid polymer electrolytes

Ionic conductivity

Decoupling of segmental dynamics
In order to design more effective solid polymer electrolytes, it is important to decouple ion conductivity from polymer segmental motion. To that end, novel polymers based on oxanorbornene dicarboximide monomers with varying lengths of oligomeric ethylene oxide side chains have been synthesized using ring opening metathesis polymerization. These unique polymers have a fairly rigid and bulky backbone and were used to investigate the decoupling of ion motion from polymer segmental dynamics. Ion conductivity was measured using broadband dielectric spectroscopy for varying levels of added lithium salt. The conductivity data demonstrate six to seven orders of separation in timescale of ion conductivity from polymer segmental motion for polymers with shorter ethylene oxide side chains. However, commensurate changes in the glass transition temperatures $\mathrm{T}_{\mathrm{g}}$ reduce the effect of decoupling in ion conductivity and lead to lower conductivity at ambient conditions. These results suggest that both, an increase in decoupling and a reduction in $T_{g}$ might be required for developing solid polymer electrolytes with high ion conductivity at room temperature.
Improving safety and performance parameters of batteries, fuel cells, and super capacitors continues to be an important focus of current research $[1,2]$. In lithium based batteries, metal dendrites can form on electrodes, pushing through the electrolyte and causing shorts, failures and fires [1, 3]. Replacement of traditional liquid electrolytes by solid electrolytes with sufficient mechanical modulus can prevent dendrite growth and improve significantly batteries safety [4-6]. One of the possible solutions could be the use of solid polymer electrolytes $[1,3]$. However, active research during the last few decades has yet to result in the development of polymer electrolytes with sufficient ionic conductivity and high mechanical modulus [7]. Despite these efforts, small molecule organic electrolytes such as ethylene carbonate and other carbonates still dominate commercial products due to their high ion conductivity. Low ion conductivity in dry polymer electrolytes at ambient conditions remains a major obstacle for use in batteries and several other applications [7].

Poly(ethylene oxide) (PEO) has long been the main material proposed as a polymer electrolyte, and serves as a benchmark for analysis of any other polymer electrolyte [8, 9]. Continued interest in PEO is also due to its unusually high
Li-salt solubility (50 wt \% of salt can be easily dissolved), and the ion conductivity reaches the required $10^{-3} \mathrm{~S} / \mathrm{cm}$ around $80^{\circ} \mathrm{C}$. However, PEO has low conductivity at room temperature, and suffers from crystallization that also severely reduces ion conductivity at ambient temperature. In addition, the transference number for Li ions in PEO is very low, $\sim 0.2$ to 0.3 [10], which likewise decreases electrolyte efficiency. In classical theories, the conductivity of ions in polymer electrolytes is primarily tied to the timescale of structural rearrangement, i.e. segmental relaxation of the polymer [11]. Attempts to improve the ionic conductivity have often focused on reducing the glass transition temperature, $\mathrm{T}_{\mathrm{g}}$, to increase the rate of segmental relaxation at ambient temperature. This approach leads to polymers with a low mechanical modulus, and still insufficient conductivity [12]. There have also been strategies to move the ethylene oxide units to the side chain of a low glass transition polymer [13-17]. This process can frustrate PEO crystallization, but the ion conductivity in these materials still remains low.

Recently a different approach in the development of potential polymer electrolytes has been emphasized [18-23].

* Corresponding authors.

E-mail addresses:waldowda@plu.edu (D. Waldow) and sokolov@utk.edu (A. Sokolov). 
This approach builds on the decoupling of ion conductivity from segmental relaxation in polymer electrolytes [23]. Creating polymer structures with frustration in chain packing opens the possibility for fast ion diffusion even when segmental dynamics are slow or frozen [17-19]. According to modern glass transition theory, frustration in chain packing also increases fragility of polymers [12, 24]. Fragility is a parameter that characterizes the steepness of the temperature dependence of segmental relaxation at temperatures close to $T_{g}[12,24]$. It has been shown that the ion motion can be decoupled from the polymer segmental dynamics in fragile polymers, and the degree of decoupling increases with an increase in fragility $[13,18,21]$. Moreover, Wojnarowska, et al., have found a decrease in the decoupling of ion conductivity from segmental dynamics under pressure in polymerized ionic liquids [25]. This result again emphasizes the importance of frustration in chain packing for the decoupling of ion conductivity. Consequently, development of polymer electrolytes with strong decoupling of ionic diffusion and sufficient salt solubility might be another promising way to design efficient solid polymer electrolytes.

In this work, we have used ring opening metathesis polymerization (ROMP) to synthesize four new polymers based on a bulky oxanorbornene dicarboxide backbone with varying oligomeric ethylene oxide (OEO) side chain lengths. These relatively rigid chains should create frustrated packing, while their chemical structure should also provide high salt solubility. Broadband dielectric spectroscopy was used to measure the ionic conductivity of lithium salt, and to estimate the degree of decoupling of conductivity from segmental dynamics. Our results indeed revealed significant decoupling of ion transport from segmental dynamics, yet, the high $\mathrm{T}_{\mathrm{g}}$ of these polymers lead to low ionic conductivity at ambient temperatures.

\section{Materials and Methods}

\subsection{Materials}

Acetonitrile (anhydrous 99.8\%,), 1-bromo-2-(2methoxyethoxy) ethane (95\%), ethyl vinyl ether $(99 \%$, ,) pentane (HPLC grade), dichloromethane (DCM, HPLC grade), ethyl acetate (99\%), pyridine (HPLC grade), bis (trifluoromethane)sulfonimide lithium salt (LiTFSI, $>99.0 \%$ ), methoxypolyethylene glycol 350 (average MW $350 \mathrm{~g} / \mathrm{mol}$ ), and Grubbs generation II catalyst were purchased from Sigma Aldrich. Furan (>99.0\%), Diethylene Glycol 2-Bromoethyl Methyl Ether (>94\%), Triethylene Glycol 2-Bromoethyl Methyl Ether (>95\%), nphenylmaleimide $(98 \%)$, and maleimide $(>98 \%)$ were purchased from TCI America, Celite ${ }^{\circledR} 545$ was from Acros Organics, and potassium carbonate (99\%) was from LaPine Scientific. All materials were used as received except for DCM which was purified further via a Glass Contour solvent purification system.
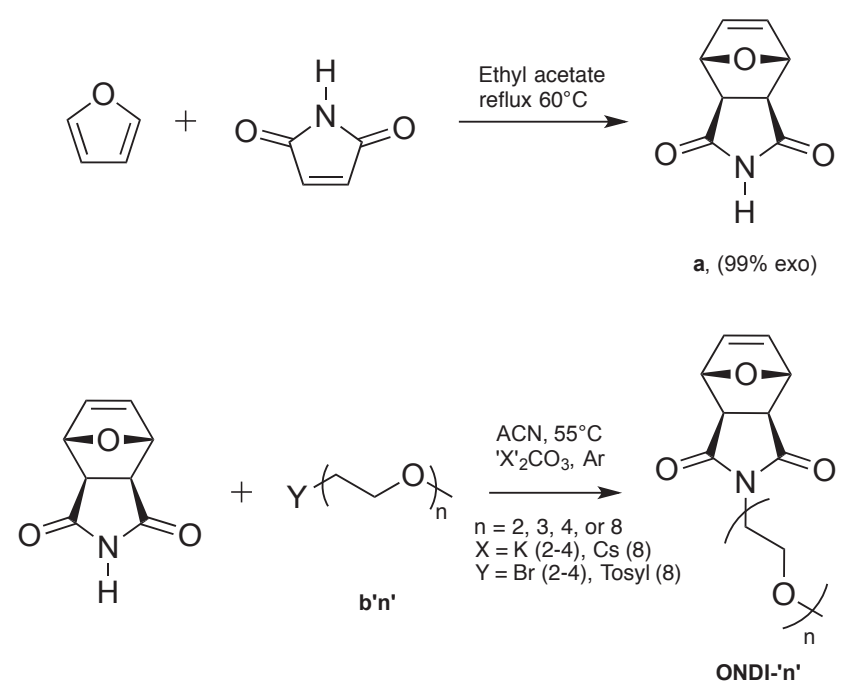

Scheme 1. Synthesis of monomers.

\subsection{Synthesis of Monomers}

The synthesis of the monomers is shown in Scheme 1 involves a Diels-Alder reaction followed by alkylation with a functionalized OEO. Only the longest OEO oligomer used a tosyl group as the leaving group while the rest used bromine.

\subsubsection{Oxanorbornene Dicarboximide (a)}

Maleimide was added to ethyl acetate in a round-bottom flask to provide an approximately $0.3 \mathrm{M}$ solution. Furan was added in a 3:1 furan to maleimide molar ratio. The resulting solution was gently refluxed for 4-6 hours and cooled. The white precipitate was vacuum filtered and dried. Typical yields of exo-7-oxabicyclo[2.2.1]hept-5-ene-2,3dicarboximide (ONDI) were $65 \%$ with a $99 \%$ exo purity measured using ${ }^{1} \mathrm{H}$ NMR.

\subsubsection{Tosylation of monofunctional oligomeric ethylene oxide}

Methoxypolyethylene glycol (average of 8 ethylene oxide units) was tosylated with $p$-toluenesulfonyl chloride following a mortar and pestle procedure adapted from Kazemi and co-workers [26]. Methoxypolyethylene glycol (34 mmol, 1.0 molar equivalent) and potassium hydroxide pellets ( $180 \mathrm{mmol}$, five molar equivalents) were added. The pellets were crushed into a granular paste after which the mixture changed color from clear to off white and became viscous. After 30 minutes, $p$-toluenesulfonyl chloride (45 mmol, 1.5 molar equivalents) and potassium carbonate (110 mmol, 3.5 molar equivalents) were slowly crushed into the paste, generating mild heat. The remaining tosyl chloride was quenched with potassium hydroxide $(140 \mathrm{mmol}, 5.0$ molar equivalents). Diethyl ether $(\sim 200 \mathrm{~mL}$ per $5 \mathrm{~g}$ of product) was used to extract the tosylated OEO, gravity filtered, and rinsed three times with diethyl ether. The solvent was removed via rotary evaporation, yielding a viscous, amber liquid (typical yield 75\%). 


\subsubsection{Alkylation of ONDI (b2, b3, and b4)}

This synthesis followed that of Al-Badri, et. al., except acetonitrile was instead of DMF [27]. Potassium carbonate, ONDI, and the relevant OEO (e.g, b3: 1-bromo-2-(2methoxyethoxy)ethane) was added under argon to a roundbottom flask in molar ratios of 1:1:3 respectively [27]. To this, approximately $60 \mathrm{~mL}$ anhydrous acetonitrile were added and the mixture was left to react at $50^{\circ} \mathrm{C}$ for $12-24$ hours. Argon atmosphere was maintained using an argonfilled balloon. The products (ONDI-'n') were adsorbed onto Celite and purified by flash chromatography with a hexane / ethyl acetate gradient, isolated, and stored under argon until use.

\subsubsection{Alkylation of ONDI (b8)}

Cesium carbonate, ONDI, and the tosylated methoxypolyethylene glycol (b8) were added under argon to a round-bottom flask in molar ratios of 6.0:1.5:1.0 respectively. To this, enough anhydrous acetonitrile was added to give a roughly $0.20 \mathrm{M}$ solution with respect to ONDI and the mixture was allowed to react at $55^{\circ} \mathrm{C}$ for 48 hours. Argon atmosphere was maintained using an argon filled balloon. The product (ONDI-8) was adsorbed onto Celite and purified by flash chromatography with ethyl acetate followed by $10 \%$ ethanol in DCM, isolated in $60 \%$ yield, and stored under argon until use.

\subsection{Grubb's Generation III Catalyst}

The synthesis of the catalyst followed the procedure of Love, and coworkers [28]. Grubb's generation II catalyst and pyridine were added to a capped flask in a $10: 1$ pyridine to catalyst molar ratio and stored under argon for five minutes. Pentane was then added in 300-fold molar excess and the mixture was allowed to rest in a freezer at $\sim-15^{\circ} \mathrm{C}$ overnight. The resulting generation III catalyst (bright green solid) was washed with pentane, vacuum filtered, and stored under argon.

\subsection{General ROMP Synthesis of Dicarboximide Polymers}

The polymers were synthesized by a ring opening metathesis polymerization (ROMP), following procedures outlined by Grubbs and co-workers [28, 29]. (Scheme 2) Flasks of monomer ( 0.9 M monomer in DCM), Grubbs catalyst with a concentration in dichloromethane appropriate for the desired degree of polymerization, and ethyl vinyl ether (1,800 molar excess) were prepared air-free under positive argon pressure. The monomer solution was added to the catalyst solution via air-free syringe techniques and allowed to react with stirring. The reaction was terminated after 18 minutes by the addition of ethyl vinyl ether. After approximately 15 minutes, the poly(ONDI-2) product was precipitated using $100 \mathrm{~mL}$ of pentane for every $10 \mathrm{~mL}$ of polymer. For polymers with OEO side chains longer than two (poly(ONDI-3, -4 , or -8$)$ ), solutions were filtered using a $0.45 \mu \mathrm{m}$ Teflon filter and the solvent was evaporated on a

Teflon sheet under argon flow followed by removal of residual solvent under vacuum.

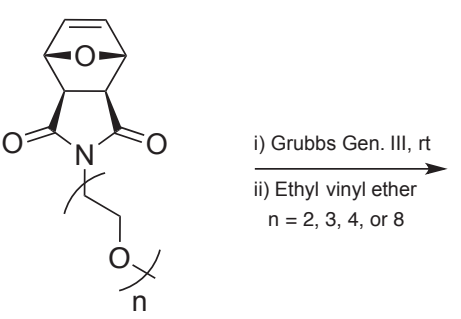

Scheme 2. Synthesis of dicarboximide based polymers.

\subsection{Sample Characterization and Separation}

Solution state nuclear magnetic resonance spectra were obtained in chloroform, except for ONDI which was dissolved in deuterated dimethylsulfoxide, using a $500 \mathrm{MHz}$ Bruker Avance III spectrometer. An Agilent PL-GPC 50 Plus with ResiPore $3 \mu \mathrm{m}$ columns was used to determine molecular weights and dispersity for the poly(ONDI-2, -3, and -4) samples using a refractive index detector relative to polystyrene standards (Agilent EasiVial) in THF while the poly(ONDI-8) sample was analyzed using Wyatt refractive index and light scattering detectors. Flash chromatography was conducted using a CombiFlash $\mathrm{R}_{\mathrm{f}}$ with normal phase RediSep $R_{f}$ silica. Relevant polymer characteristics are presented in Table 1.

\subsection{Ionic Conductivity Measurements}

The polymer electrolytes were prepared by dissolving polymers and appropriate amounts of LiTFSI salt in acetone, and subsequently removing the solvent in a vacuum oven at room temperature. The samples were further dried at $80^{\circ} \mathrm{C}$ for at least three days. Broadband dielectric spectroscopy measurements of the polymer electrolytes were carried out using a Novocontrol alpha analyzer with ZGS interface in the frequency range of $10^{-2}-10^{7} \mathrm{~Hz}$. The temperature was controlled by a Novocontrol Quatro cryosystem, with temperature stability of $\pm 0.1 \mathrm{~K}$. The polymer electrolyte sample under test was sandwiched between gold-plated electrodes with a $10 \mathrm{~mm}$ diameter and a Teflon spacer of $0.054 \mathrm{~mm}$ thickness. The spacer defines the thickness of the samples. The samples were additionally annealed in the sample cell in dry nitrogen atmosphere at the highest experimental temperature for approximately 30 minutes prior to the dielectric measurement.

\subsection{DSC Measurements}

Differential scanning calorimetry (DSC) measurements were performed using TA Q2000 instrument and Perkin-Elmer Pyris 1 instruments. No signs of crystallization have been detected on heating and/or cooling cycles for all samples studied. $\mathrm{T}_{\mathrm{g}}$ has been estimated on cooling during the second cycle of heating and cooling (to remove any thermal history), 
with the cooling rate $10 \mathrm{~K} / \mathrm{min}$. $\mathrm{T}_{\mathrm{g}}$ was taken as the midpoint of the specific heat step associated with the glass transition.
The obtained thermal data and other sample characteristics are presented in Table 1.

\section{Table 1}

Structural information is presented for the synthesized polymer electrolytes and includes: name, abbreviation, molecular weight $\left(\mathrm{M}_{\mathrm{w}}\right)$, dispersity $(Ð)$, weight percent of LiTFSI used, and the glass transition temperature $\left(\mathrm{T}_{\mathrm{g}}\right)$ estimated from DSC. VFT parameters are obtained from the fit of temperature dependence of segmental dynamics in neat polymers (Fig. 2) and from the fits of temperature dependence of conductivity in the case of polymers with salt (Fig. 4). Fragility (m) for the neat polymers is estimated from the temperature dependence of their segmental relaxation.

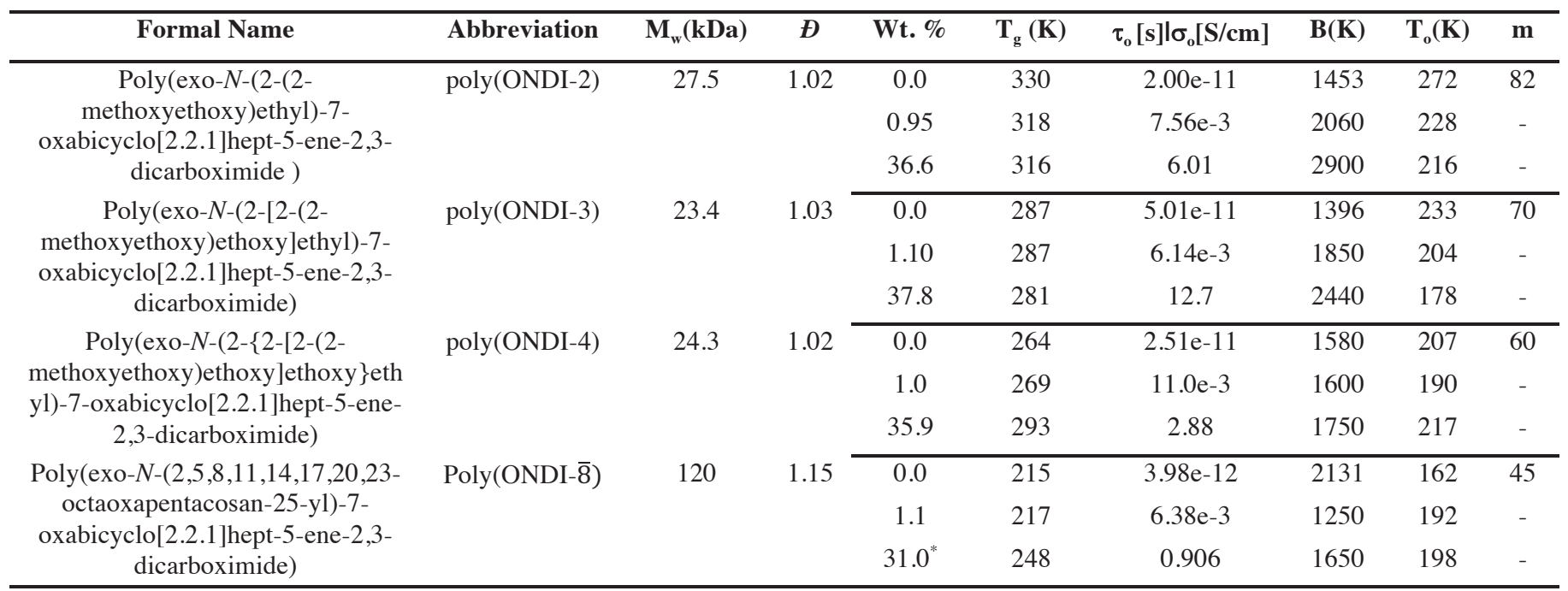

*The glass transition temperature was measured at $30.0 \mathrm{wt}$ \% for this sample.

\section{Results}

The dielectric loss spectra of the neat polymers show a peak that corresponds to segmental relaxation, and a tail of conductivity at lower frequency (Fig. 1). The frequency of the peak maximum provides estimates of the segmental

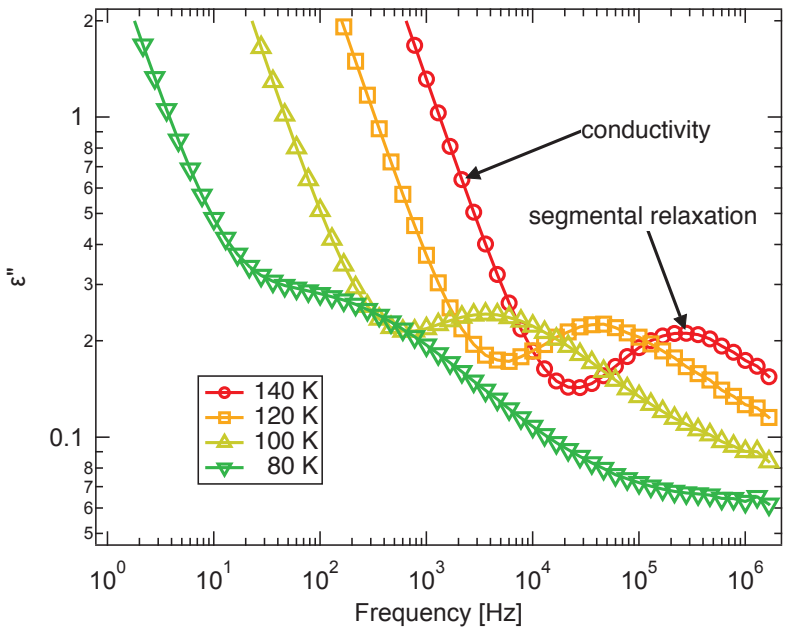

Fig. 1. The loss spectra, $\varepsilon$ "(f) of neat poly(ONDI-2) as a function of frequency at a few selected temperatures, show both segmental relaxation and conductivity.

relaxation time $\tau_{\mathrm{S}}(\mathrm{T})$ (Fig. 2). The data show that an increase in the OEO side chain length leads to faster segmental dynamics as is also seen by a decrease in the glass transition temperature $\mathrm{T}_{\mathrm{g}}$. Unfortunately, the high ionic conductivity contribution covers the segmental relaxation peak in polymers with salt, preventing the analysis of segmental relaxation time in these samples.

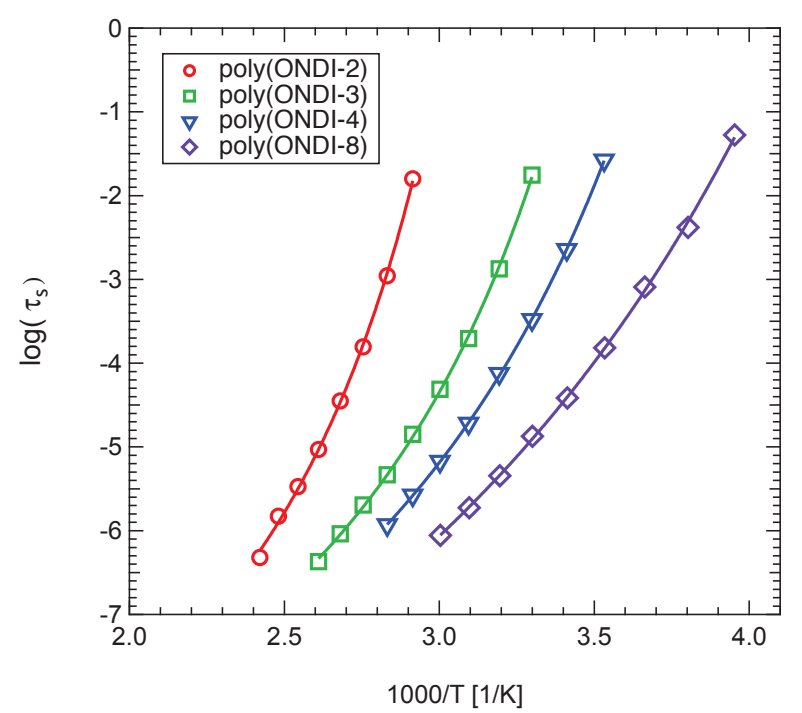

Fig. 2. Segmental relaxation times in neat polymers (symbols) versus inverse temperature. The solid lines present VFT fits (Eq. 1).

Examples of the conductivity spectra as a function of frequency are shown in Fig. 3 for poly(ONDI-4) with $36 \%$ LiTFSI for temperatures from $90{ }^{\circ} \mathrm{C}$ to $10{ }^{\circ} \mathrm{C}$. The conductivity spectra show the usual three regimes: $\mathrm{AC}$ conductivity at high frequency; a frequency independent DC 
conductivity $\sigma(\mathrm{T})$ regime at intermediate frequency; and a sharp decrease due to electrode polarization effects at low frequencies. A decrease in temperature leads to a decrease in conductivity and to shift of the characteristic frequencies to

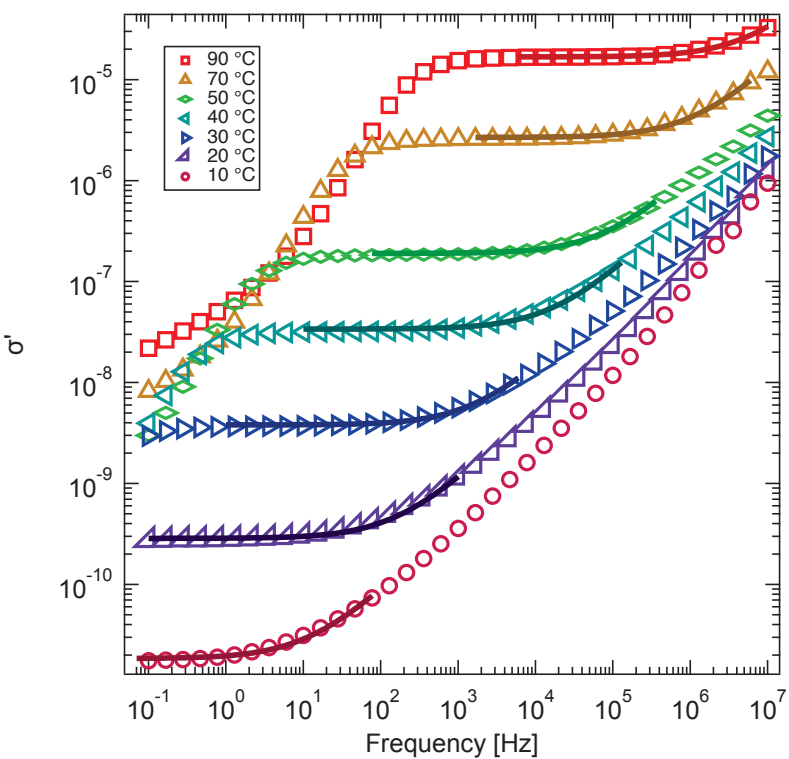

Fig. 3. The logarithm of conductivity $(\mathrm{S} / \mathrm{cm})$ as a function of frequency (symbols) for poly(ONDI-4) with 36\% LiTFSI at several temperatures. The plateau in the intermediate frequency range presents DC conductivity at that temperature. The solid lines present fits to the RBM model (Eq. 2).

the lower values due to slowing down of ion diffusion. These spectra provide a direct measure of ionic conductivity at different temperatures for samples with different salt contents.

Fig. 4 presents a plot of the logarithm of conductivity as a function of temperature for all polymers with a $1 \%$ LiTFSI (Fig. 2a) and an $\sim 35 \%$ LiTFSI (Fig. 2b) salt concentration with PEO data from Edman, et al. [30]. The data generally show that for both salt concentrations an increase in the OEO side chain length leads to a significant increase in ion conductivity. The primary cause of this increase in conductivity is the significant reduction of the glass transition temperature (shown by arrows in the Fig. 2b). Our analysis reveals that adding LiTFSI salt increases $T_{g}$ in polymers with longer OEO side chains ( $n=4$ and 8$)$. However, this trend reverses in the polymers with short $(n=2-3)$ OEO side chains (Table 1).

\section{Discussion}

Our polymers are good glass-forming systems, and no sign of crystallization has been detected in the DSC measurements. The $\mathrm{T}_{\mathrm{g}}$ of the neat polymers decreases with an increase in the OEO side chain length (Table 1). This is caused by a plasticizing effect that the flexible OEO groups provide for the polymer. The segmental relaxation time in all neat polymers exhibit typical Vogel-Fulcher-Tammann (VFT) behavior:
$\tau_{S}=\tau_{0} \exp \left(\frac{B}{T-T_{0}}\right)$

The temperature dependence of segmental relaxation was fit to Eq.1 (Fig. 2) and the VFT fit parameters are presented in Table 1. Using the VFT parameters, the steepness of the temperature variations of segmental dynamics were estimated with the so-called fragility parameter, $\mathrm{m}=\mathrm{BT}_{\mathrm{g}} /\left[\ln 10 *\left(\mathrm{~T}-\mathrm{T}_{\mathrm{g}}\right)^{2}\right] \quad[24]$. The fragility parameter decreases with an increase in the length of the OEO side chain (Table 1). This result is expected because flexible OEO side chains improve packing of polymer chains, and the latter, according to Generalized Entropy Theory [24, 31], leads to a decrease in fragility.

Usually the addition of salt increases $T_{g}$ of polymer electrolytes (e.g. in PPG [15]). The same was observed for our polymers with longer OEO side chains $(n=4$ and $n=8)$. Surprisingly, $\mathrm{T}_{\mathrm{g}}$ decreases in the case of the polymers with shorter OEO side chain lengths $(n=2$ and $n=3)$. Especially
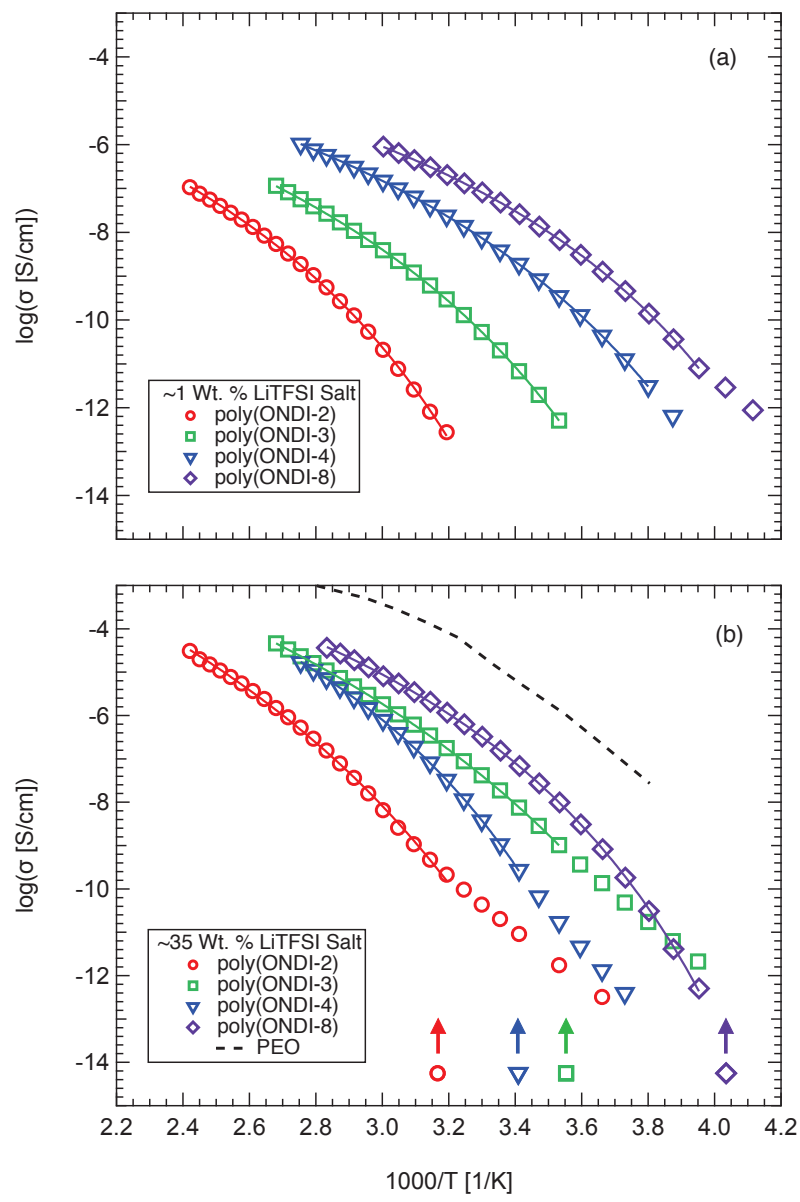

Fig. 4. Temperature dependence of the logarithm of DC conductivity (symbols) for polymer samples with 1 wt $\%$ of LiTFSI salt (a); and approximately $35 \mathrm{wt} \%$ of LiTFSI salt (b). Arrows in (b) mark $\mathrm{T}_{\mathrm{g}}$ measured by DSC for the high salt containing samples, and the black dashed line presents conductivity data for PEO (data from [30]). The solid lines present fits to the VFT function. 
surprising is that at high salt concentration $\mathrm{T}_{\mathrm{g}}$ of the polymer with $n=3$ appears a bit lower than $T_{g}$ of the polymer with $n=4$. We don't have an explanation for these observations. These results also show rather high solubility of LiTFSI salt in the polymers presumably due to both the OEO side chains as well as the backbone structure.
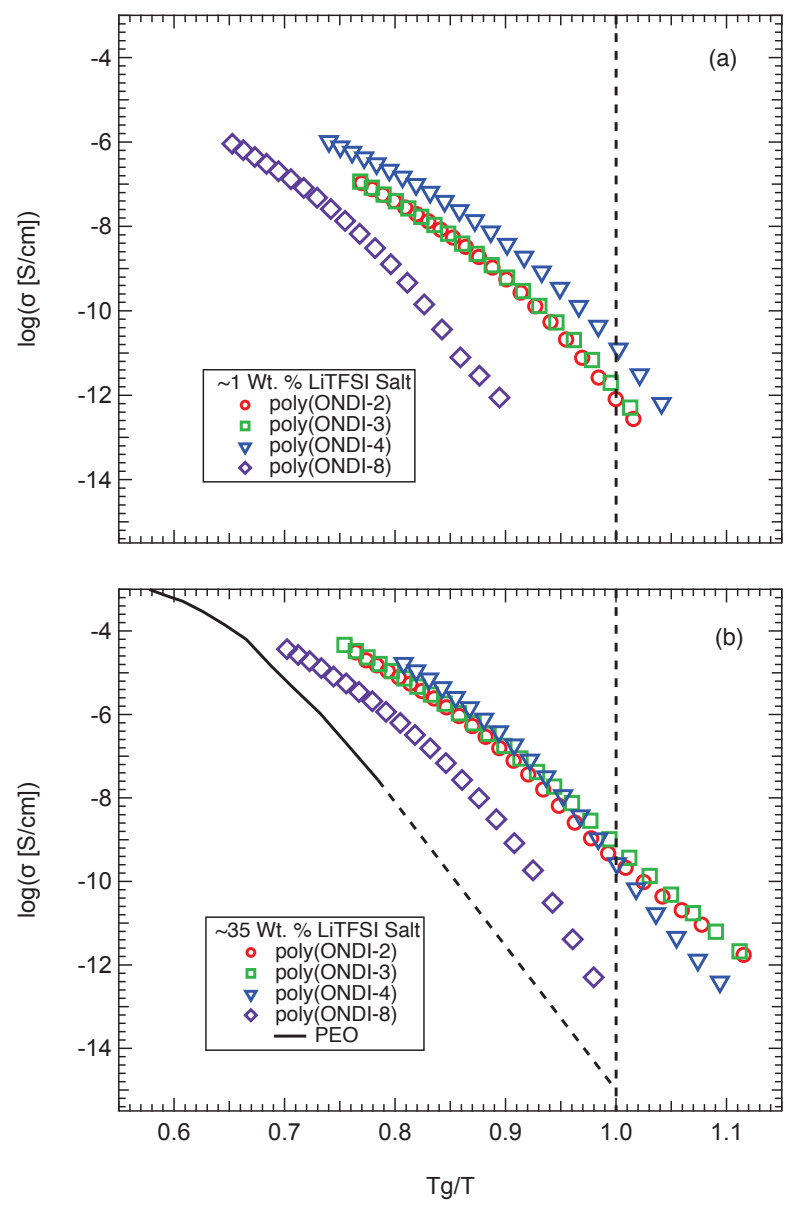

Fig. 5. The logarithm of conductivity versus inverse temperature scaled by $\mathrm{T}_{\mathrm{g}}$ for all four polymers with $1 \mathrm{wt} \%$ LiTFSI salt (a) and $\sim 35 \mathrm{wt} \%$ LiTFSI salt (b). Conductivity at $T_{g}$ is significantly higher than $10^{-14}-10^{-15} \mathrm{~S} / \mathrm{cm}$, indicating strong decoupling of ion conductivity from segmental dynamics. The solid line shows the data for PEO, and the dashed line is an extrapolation to $\mathrm{T}_{\mathrm{g}}$. The vertical dashed lines indicate the $\mathrm{T}_{\mathrm{g}}$ in the $\mathrm{x}$-axis.

Analysis of the conductivity data clearly demonstrates that a decrease in $\mathrm{T}_{\mathrm{g}}$ is the major factor that leads to an increase in conductivity at ambient conditions. However, none of the four polymers provides conductivity at room temperature comparable to the conductivity of PEO with suppressed crystallization (Fig. 4b). The reason appears to be the same: the $\mathrm{T}_{\mathrm{g}}$ of PEO with salt is still lower than the $\mathrm{T}_{\mathrm{g}}$ of our polymers with salt. Conductivity data in the samples at $\mathrm{T}>\mathrm{T}_{\mathrm{g}}$ show traditional VFT behavior (Fig. 4), and the VFT fit parameters are presented in the Table 1.

To analyze the decoupling in these samples, the role of $\mathrm{T}_{\mathrm{g}}$ can be removed by presenting the conductivity data versus temperature scaled to $\mathrm{T}_{\mathrm{g}}\left(\mathrm{T}_{\mathrm{g}} / \mathrm{T}\right)$ (Fig. 5). For ion motion that is well coupled to structural relaxation and good ion pair dissociation, conductivity at $\mathrm{T}_{\mathrm{g}}$ for ionic liquids should be $\sim 10^{-14}-10^{-15} \mathrm{~S} / \mathrm{cm}[32,33]$. In the case of polymers, electrolyte conductivity at $\mathrm{T}_{\mathrm{g}}$ also depends on the volume fraction of the salt. Hence, the conductivity at $\mathrm{T}_{\mathrm{g}}$ should be proportional to the salt concentration. So, for a $30 \mathrm{wt} \%$ salt sample with good salt dissociation and strong coupling of ion and segmental motions, the conductivity at $\mathrm{T}_{\mathrm{g}}$ is expected to be on the order of $\sim 0.3^{*}\left(10^{-14}-10^{-15}\right) \mathrm{S} / \mathrm{cm}$.

Our data reveal much higher conductivities at $\mathrm{T}_{\mathrm{g}}\left(\sim 10^{-9}\right.$ $\mathrm{S} / \mathrm{cm})$ for polymers with shorter OEO side chains $(\mathrm{n}=2-4)$ as

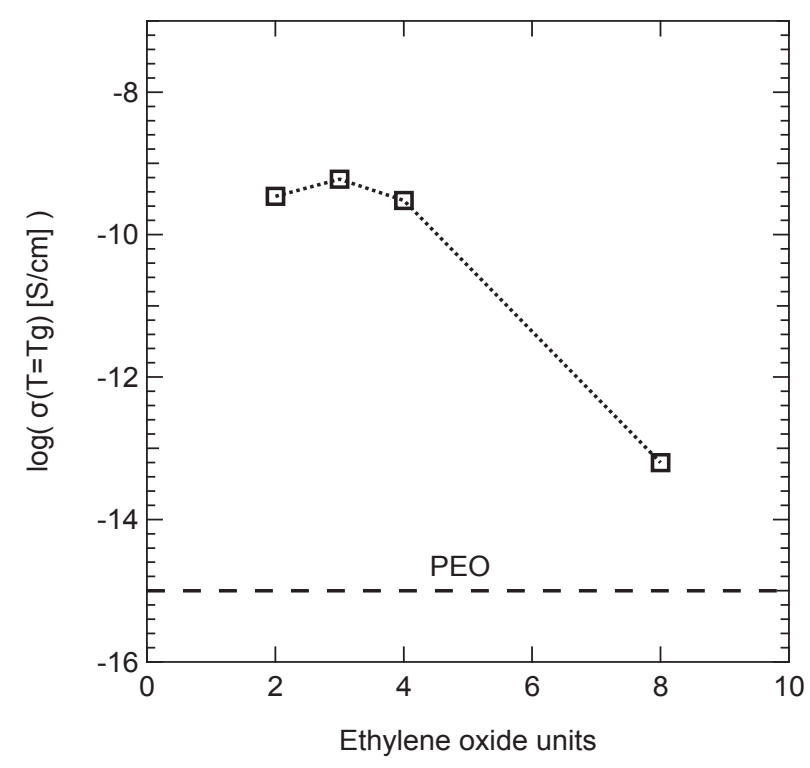

Fig. 6. The logarithm of conductivity at the glass transition temperature for all four polymer samples with $35 \mathrm{wt} \%$ LiTFSI salt as a function of the OEO side chain length.

shown in Fig. 6. This result clearly indicates a strong ( 5-6 orders of magnitude) decoupling of ion diffusion from segmental dynamics in our polymers with shorter OEO side chains. Thus, the presented analysis indeed confirms our initial hypothesis that constructing rigid polymers with frustrated chain packing will lead to a decoupling of ion motion from segmental relaxation.

However, the obtained estimates of the decoupling of ion transport from segmental dynamics using conductivity at $\mathrm{T}_{\mathrm{g}}$ assumes that all ions are contributing to conductivity. This is usually not the case in polymer electrolytes [22, 34, 35]. To estimate the fraction of ions contributing to the measured ionic conductivity, a recently developed model [36] was used. This model relates the conductivity relaxation time, $\tau_{\sigma}$, to the ion diffusion on a distance comparable to the average distance between ion with different charges, $R=\left[3 /\left(4 \pi n_{p}\right)\right]^{1 / 3}$, where $n_{p}$ is the concentration of ion pairs (salt) in the sample. The model ascribes the conductivity relaxation to a reorientation of a dipole formed by an ion pair [36]. The 
conductivity relaxation time can be estimated using Random Barrier Model (RBM) [37] that describes the conductivity spectra in the crossover region from DC to AC conductivity (Fig. 3):

$\sigma(\omega)=\sigma_{0}\left[\frac{i \omega \tau_{\sigma}}{\ln \left(1+i \omega \tau_{\sigma}\right)}\right]$

The diffusion coefficient of ions, D, can then be estimated using $\tau_{\sigma}$ in Eq. 3:

$\mathrm{D}=\lambda^{2} / 6 \tau_{\sigma}$

where $\lambda$ is a characteristic distance ions need to diffuse to reorient the dipole [36]. In the case of both ions moving with similar rate, $\lambda \approx \mathrm{R} / 2[36]$.
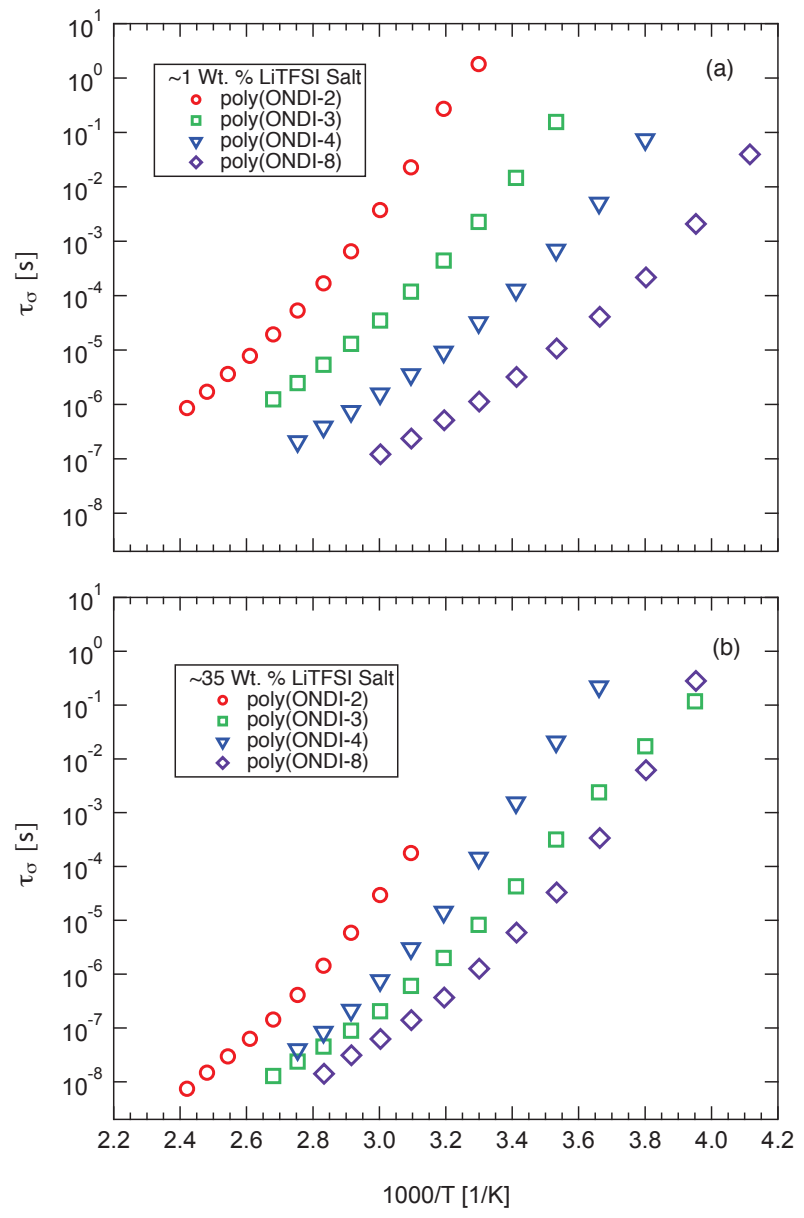

Fig. 7. Conductivity relaxation times, $\tau_{\sigma}$, for all polymers at low salt (a) and high salt (b) samples are presented versus inverse temperature.

Following this model, the conductivity spectra are first fit to the RBM Eq. 2 (Fig.3). The obtained $\tau_{\sigma}(\mathrm{T})$ for all the samples with salt are presented in the Fig. 7. To calculate the diffusion coefficients, mass densities of these samples need to be estimated as they are not known. However, the density of polymer electrolytes with salt is usually in the range 1-1.6 $\mathrm{g} / \mathrm{cm}^{3}$, and using any number in this range will not affect the estimates significantly. So, we assumed the density to be in the middle of this range, $\sim 1.3 \mathrm{~g} / \mathrm{cm}^{3}$, and estimated the concentration of ion pairs $n_{p}$ taking into account the wt $\%$ of salt in the samples. With these values of $n_{p}$, the diffusion coefficients of ions were estimated for our samples (Fig. 8).

The 'effective' concentration of ions contributing to conductivity, $\mathrm{n}^{*}$, was then estimated making use of the obtained diffusion coefficients in the Nerst-Einstein equation for conductivity:

$\sigma=\frac{q^{2} D}{k T} n^{*}$

where $q$ is the ion charge. Assuming that both ions contribute to conductivity, the degree of ion dissociation, taken as the ratio $n^{*}(T) /\left(2 * n_{p}\right)$, was estimated (Fig. 9). This analysis reveals that degree of ion dissociation is a bit higher in polymers with lower salt content. Moreover, as expected, the increase in the length of OEO side chain improves salt
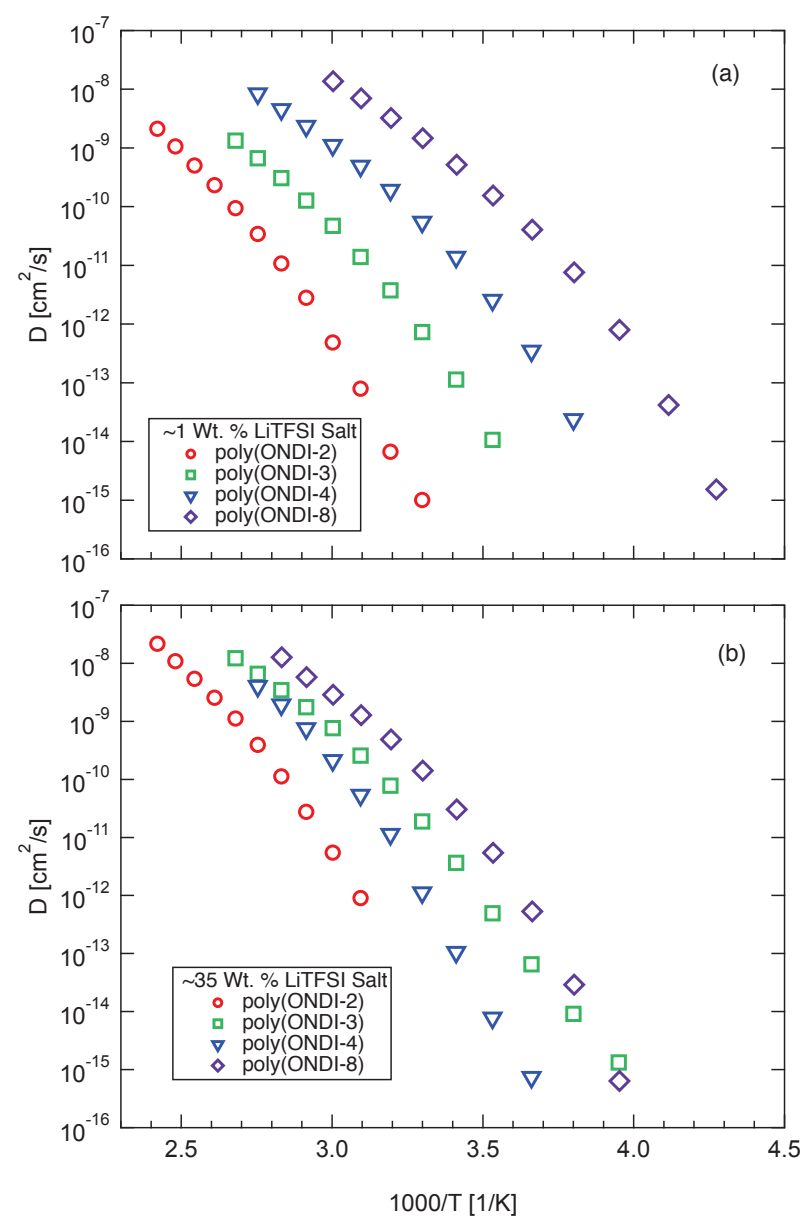

Fig. 8. Estimated diffusion coefficients for all polymers at low salt (a) and high salt (b) samples are presented versus inverse temperature.

dissociation at the same ion concentration (Fig. 9). These results clearly demonstrate that only about $10 \%$ of the ions contribute to the measured conductivity at $\mathrm{T}_{\mathrm{g}}$ (Fig. 9). Thus, the decoupling of ion conductivity at $\mathrm{T}_{\mathrm{g}}$ is actually $\sim 10$ times higher than estimated using just $\sigma\left(\mathrm{T}_{\mathrm{g}}\right)$ (Fig. 6). 
The presented data suggest that at $\mathrm{T}_{\mathrm{g}}$ the rate of ion diffusion is 6-7 orders faster than the rate of segmental relaxation in polymers with short OEO side chains. It is a significant decoupling of ion motion from segmental dynamics, suggesting that ions make millions of jumps on the time scale of a single segmental relaxation event. Nevertheless, these rigid chains also have higher $\mathrm{T}_{\mathrm{g}}$, and the latter leads to lower conductivity at room temperature. The increase in the length of the OEO side chains with $n>4$ leads to a decrease in $T_{g}$, but also a decrease in decoupling (Fig. 6). The latter is expected $[18,21,22]$ due to the decrease in frustration of chain packing evident in much lower fragility (Table 1).

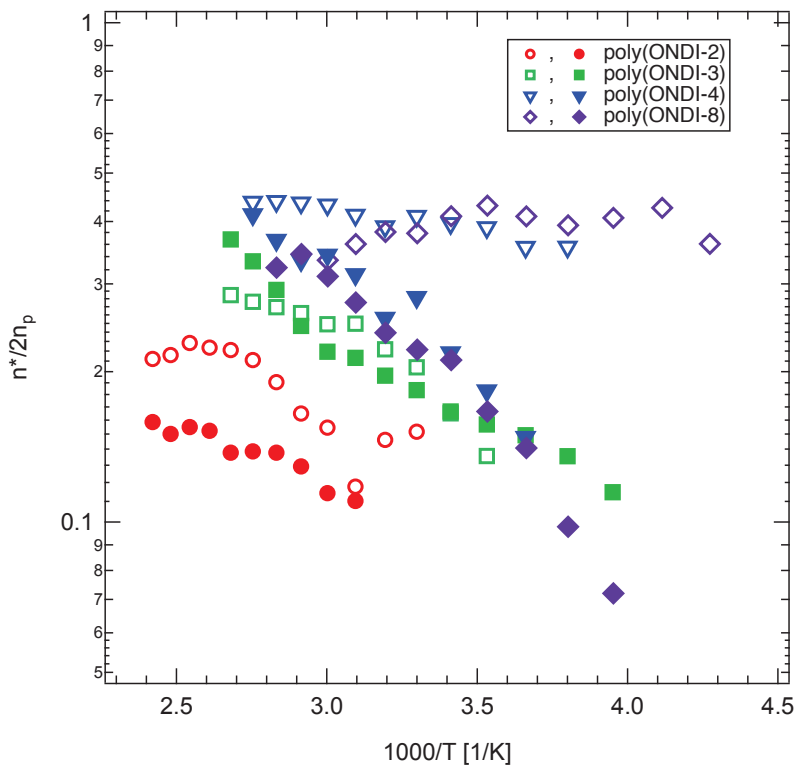

Fig. 9. The estimated salt disassociation for all polymers is plotted as a function of inverse temperature. The open and closed symbols represent the low and high concentration salt samples, respectively.

Moreover, the conductivity at room temperature increases emphasizing more important role of $\mathrm{T}_{\mathrm{g}}$ for these polymers. Therefore, it is important to create polymer electrolytes with increased chain packing frustration while still maintaining a low $\mathrm{T}_{\mathrm{g}}$. It is known that there are low $\mathrm{T}_{\mathrm{g}}$ polymers with relatively high fragility (e.g. PDMS) [24, 38], suggesting that it should be possible to combine frustration in chain packing with low $\mathrm{T}_{\mathrm{g}}$.

\section{Conclusion}

We successfully synthesized polymers with a relatively rigid backbone and OEO side groups of different lengths. The latter were introduced to provide high $\mathrm{Li}$ salt solubility. Rigidity of the backbone led to a frustration in chain packing and, as a consequence, significant decoupling of ionic conductivity from segmental dynamics. The decoupling reaches $\sim 6-7$ orders of magnitude in polymers with shorter OEO side chains.

However, the synthesized polymers have relatively high $\mathrm{T}_{\mathrm{g}}$ that decreases with the increase in OEO side chain length.
Unfortunately, the decoupling of ionic conductivity was also decreasing with the increase in OEO side chain length. This can be caused by a decrease in chain packing frustration due to flexible OEO side groups (evident in the decrease of the neat polymer fragility), and also to strong coordination of $\mathrm{Li}$ ions to oxygen in the side groups, which is well-known for PEO. Nonetheless, the decrease in $\mathrm{T}_{\mathrm{g}}$ has a larger effect on conductivity than the decrease in decoupling, and so samples with longer OEO side chains have higher ion conductivity at room temperature. Accordingly, we need to search for low$\mathrm{T}_{\mathrm{g}}$ polymers that will have increased frustration in chain packing and strong decoupling of ion conductivity from segmental dynamics. It remains uncertain whether this is possible, although there are no physical principles preventing combination of these effects.

\section{Acknowledgements}

DAW acknowledges National Science Foundation awards (RUI: DMR-1411247 and MRI-07233226), the H. Eugene LeMay, Jr. and Robert C. Olsen funds, Dr. N. Yakelis for helpful synthetic discussions, and Dr. A. J. Boydston and R. Zhuravlev for additional GPC and DSC measurements, respectively. APS acknowledges financial support by the U.S. Department of Energy, Office of Science, Basic Energy Sciences, Materials Science \& Engineering Division.

\section{References}

[1] J.B. Goodenough and Y. Kim, Challenges for Rechargeable Li Batteries, Chemistry of Materials 22 (2010) 587-603.

[2] V. Etacheri, R. Marom, R. Elazari, G. Salitra, and D. Aurbach, Challenges in the development of advanced Li-ion batteries: a review, Energy \& Environmental Science 4 (2011) 3243-3262.

[3] V. Di Noto, S. Lavina, G.A. Giffin, E. Negro, and B. Scrosati, Polymer electrolytes: Present, past and future, Electrochimica Acta 57 (2011) 4-13.

[4] M.D. Tikekar, S. Choudhury, Z. Tu, and L.A. Archer, Design principles for electrolytes and interfaces for stable lithium-metal batteries, Nature Energy 1 (2016) 16114.

[5] K.J. Harry, D.T. Hallinan, D.Y. Parkinson, A.A. MacDowell, and N.P. Balsara, Detection of subsurface structures underneath dendrites formed on cycled lithium metal electrodes, Nat Mater 13 (2014) 69-73.

[6] S.A. Mullin, G.M. Stone, A. Panday, and N.P. Balsara, Salt Diffusion Coefficients in Block Copolymer Electrolytes, Journal of The Electrochemical Society 158 (2011) A619.

[7] M. Singh, O. Odusanya, G.M. Wilmes, H.B. Eitouni, E.D. Gomez, A.J. Patel, V.L. Chen, M.J. Park, P. Fragouli, H. Iatrou, N. Hadjichristidis, D. Cookson, and N.P. Balsara, Effect of Molecular Weight on the Mechanical and Electrical Properties of Block 
Copolymer Electrolytes, Macromolecules 40 (2007) 4578-4585.

[8] M. Armand, The history of polymer electrolytes, Solid State Ionics 69 (1994) 309-319.

[9] D.E. Fenton, J.M. Parker, and P.V. Wright, Complexes of alkali metal ions with poly(ethylene oxide), Polymer 14 (1973) 589.

[10] K. Hayamizu, E. Akiba, T. Bando, and Y. Aihara, $1 \mathrm{H}, 7 \mathrm{Li}$, and $19 \mathrm{~F}$ nuclear magnetic resonance and ionic conductivity studies for liquid electrolytes composed of glymes and polyetheneglycol dimethyl ethers of $\mathrm{CH} 3 \mathrm{O}(\mathrm{CH} 2 \mathrm{CH} 2 \mathrm{O}) \mathrm{nCH} 3(\mathrm{n}=3-50)$ doped with $\mathrm{LiN}(\mathrm{SO} 2 \mathrm{CF}$ )2, The Journal of Chemical Physics 117 (2002) 5929-5939.

[11] M.A. Ratner and D.F. Shriver, Ion transport in solventfree polymers, Chemical Reviews 88 (1988) 109-124.

[12]Z. Xue, D. He, and X. Xie, Poly(ethylene oxide)-based electrolytes for lithium-ion batteries, Journal of Materials Chemistry A 3 (2015) 19218-19253.

[13] F. Fan, Y. Wang, T. Hong, M.F. Heres, T. Saito, and A.P. Sokolov, Ion Conduction in Polymerized Ionic Liquids with Different Pendant Groups, Macromolecules 48 (2015) 4461-4470.

[14] J. Bartels, A. Hess, H.-S. Shiau, H.R. Allcock, R.H. Colby, and J. Runt, Synthesis, Morphology, and Ion Conduction of Polyphosphazene Ammonium Iodide Ionomers, Macromolecules 48 (2015) 111-118.

[15] J. Sun, G.M. Stone, N.P. Balsara, and R.N. Zuckermann, Structure-Conductivity Relationship for Peptoid-Based PEO-Mimetic Polymer Electrolytes, Macromolecules 45 (2012) 5151-5156.

[16] P.M. Blonsky, D.F. Shriver, P. Austin, and H.R. Allcock, Polyphosphazene solid electrolytes, Journal of the American Chemical Society 106 (1984) 6854-6855.

[17]H.R. Allcock, J.D. Bender, R.V. Morford, and E.B. Berda, Synthesis and Characterization of Novel Solid Polymer Electrolytes Based on Poly(7-

oxanorbornenes) with Pendent OligoethyleneoxyFunctionalized Cyclotriphosphazenes, Macromolecules 36 (2003) 3563-3569.

[18] A.L. Agapov and A.P. Sokolov, Decoupling Ionic Conductivity from Structural Relaxation: A Way to Solid Polymer Electrolytes?, Macromolecules 44 (2011) 4410-4414.

[19] F. Fan, Y. Wang, and A.P. Sokolov, Ionic Transport, Microphase Separation, and Polymer Relaxation in Poly(propylene glycol) and Lithium Perchlorate Mixtures, Macromolecules 46 (2013) 9380-9389.

[20] P. Griffin, A.L. Agapov, A. Kisliuk, X.-G. Sun, S. Dai, V.N. Novikov, and A.P. Sokolov, Decoupling charge transport from the structural dynamics in room temperature ionic liquids, The Journal of Chemical Physics 135 (2011) 114509-114508.

[21] Y. Wang, A.L. Agapov, F. Fan, K. Hong, X. Yu, J. Mays, and A.P. Sokolov, Decoupling of Ionic Transport from Segmental Relaxation in Polymer
Electrolytes, Physical Review Letters 108 (2012) 088303.

[22] Y. Wang, F. Fan, A.L. Agapov, T. Saito, J. Yang, X. Yu, K. Hong, J. Mays, and A.P. Sokolov, Examination of the fundamental relation between ionic transport and segmental relaxation in polymer electrolytes, Polymer 55 (2014) 4067-4076.

[23] Y. Wang and A.P. Sokolov, Design of superionic polymer electrolytes, Current Opinion in Chemical Engineering 7 (2015) 113-119.

[24] K. Kunal, C.G. Robertson, S. Pawlus, S.F. Hahn, and A.P. Sokolov, Role of Chemical Structure in Fragility of Polymers: A Qualitative Picture, Macromolecules 41 (2008) 7232-7238.

[25]Z. Wojnarowska, J. Knapik, J. Jacquemin, S. Berdzinski, V. Strehmel, J.R. Sangoro, and M. Paluch, Effect of Pressure on Decoupling of Ionic Conductivity from Segmental Dynamics in Polymerized Ionic Liquids, Macromolecules 48 (2015) 8660-8666.

[26] F. Kazemi, A.R. Massah, and M. Javaherian, Chemoselective and scalable preparation of alkyl tosylates under solvent-free conditions, Tetrahedron 63 (2007) 5083-5087.

[27] Z.M. Al-Badri and G.N. Tew, Well-Defined Acetylene-Functionalized Oxanorbornene Polymers and Block Copolymers, Macromolecules 41 (2008) 4173-4179.

[28] J.A. Love, J.P. Morgan, T.M. Trnka, and R.H. Grubbs, A practical and highly active ruthenium-based catalyst that effects the cross metathesis of acrylonitrile, Angewandte Chemie, International Edition 41 (2002) 4035-4037.

[29] T.-L. Choi and R.H. Grubbs, Controlled living ringopening-metathesis polymerization by a fast-initiating ruthenium catalyst, Angewandte Chemie, International Edition 42 (2003) 1743-1746.

[30] L. Edman, A. Ferry, and M.M. Doeff, Slow recrystallization in the polymer electrolyte system poly(ethylene oxide)n-LiN(CF3SO2)2, Journal of Materials Research 15 (2000) 1950-1954.

[31] J. Dudowicz, K.F. Freed, and J.F. Douglas, Entropy theory of polymer glass formation revisited. I. General formulation, The Journal of Chemical Physics 124 (2006) 064901.

[32] J.R. Sangoro, A. Serghei, S. Naumov, P. Galvosas, J. Kärger, C. Wespe, F. Bordusa, and F. Kremer, Charge transport and mass transport in imidazolium-based ionic liquids, Physical Review E 77 (2008) 051202.

[33] J.R. Sangoro, C. Iacob, A.L. Agapov, Y. Wang, S. Berdzinski, H. Rexhausen, V. Strehmel, C. Friedrich, A.P. Sokolov, and F. Kremer, Decoupling of ionic conductivity from structural dynamics in polymerized ionic liquids, Soft Matter 10 (2014) 3536-3540.

[34] R.J. Klein, S. Zhang, S. Dou, B.H. Jones, R.H. Colby, and J. Runt, Modeling electrode polarization in dielectric spectroscopy: Ion mobility and mobile ion 
concentration of single-ion polymer electrolytes, The Journal of Chemical Physics 124 (2006) 144903.

[35] Y. Wang, C.-N. Sun, F. Fan, J.R. Sangoro, M.B.

Berman, S.G. Greenbaum, T.A. Zawodzinski, and A.P. Sokolov, Examination of methods to determine freeion diffusivity and number density from analysis of electrode polarization, Physical Review E 87 (2013) 042308 .

[36] C. Gainaru, E.W. Stacy, V. Bocharova, M. Gobet, A.P. Holt, T. Saito, S. Greenbaum, and A.P. Sokolov, Mechanism of Conductivity Relaxation in Liquid and Polymeric Electrolytes: Direct Link between
Conductivity and Diffusivity, The Journal of Physical Chemistry B 120 (2016) 11074-11083.

[37] J.C. Dyre, A simple model of ac hopping conductivity in disordered solids, Physics Letters A 108 (1985) 457461.

[38] Q. Qin and G.B. McKenna, Correlation between dynamic fragility and glass transition temperature for different classes of glass forming liquids, Journal of Non-Crystalline Solids 352 (2006) 2977-2985. 